\title{
Variant of Demodex folliculorum infesting the eyelids
}

\author{
F. P. ENGLISH
}

Brisbane, Australia

The mite Demodex folliculorum, which is currently evoking interest amongst ophthalmologists throughout the world, has been thoroughly described in detail by Coston (1967), Smith and McGulloch ( I969), and English (r 969).

Mites and ticks, in contradistinction to the other arachnids have fused segments, giving a characteristic morphological appearance. Accordingly, the adult type of Demodex folliculorum presents a vermiform or cigar-shaped profile (Fig. I) with a well differentiated prosoma incorporating head and thorax, and an opisthosoma consisting of abdomen and tail ending in a smoothly rounded off caudal extremity. The segmented thorax bears four paired appendages and the abdomen is distinguished by annular markings or grooves. This latter feature possibly is a type of dermatoglyphic adaptation to its follicular environment, allowing for its facility of ready ingress or egress.

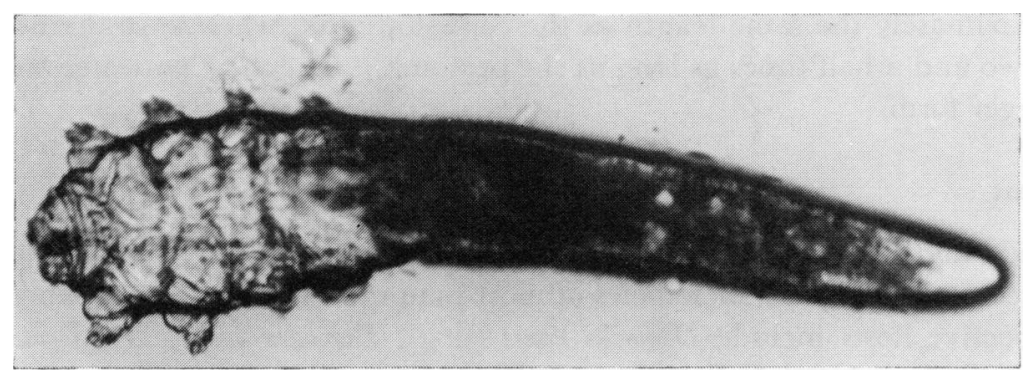

F I G. I Ventral view of adult specimen of Demodex folliculorum. $\times 400$

(Courtesy of The Medical Journal of Australia: English, 1969)

A variant of Demodex folliculorum can sometimes be found in the eyelids, and this may lead to confusion in identification of the infesting organism and a mistaken diagnosis.

\section{Methods and materials}

In reviewing an elderly male patient suffering with blepharitis parasitica of demodectic aetiology, an unusual variant was observed when the eyelashes mounted in a peanut oil medium were subjected to microscopic examination.

\section{Observations}

One microscopic slide (Fig. 2, overleaf) displayed the whole range of the life history of the parasite passing from the egg through the immature to the adult stage and also contained the interesting caudate variant, located near the bulb of the eyelash. 
(2)

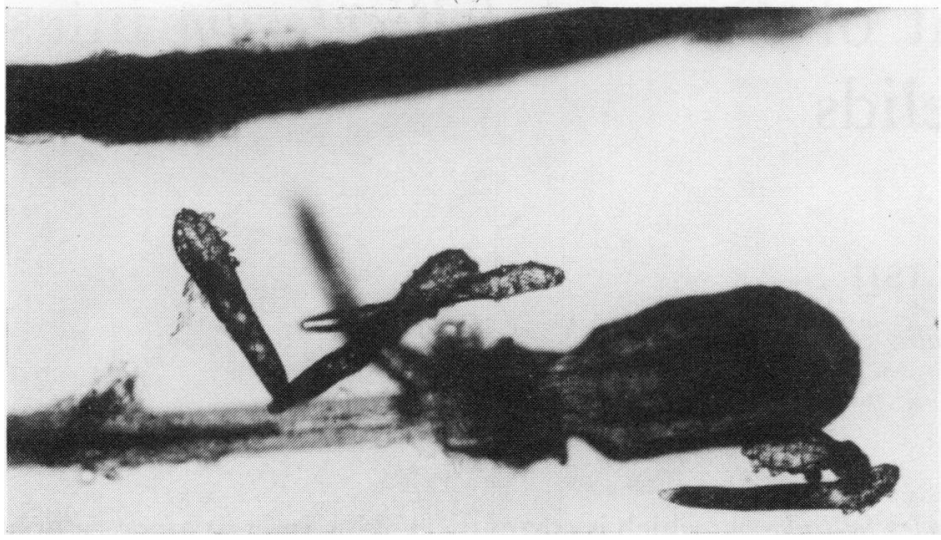

FIG. 2 Range of presentation of Demodex folliculorum, the egg, and the immature and adult stages. The short variety is located near the bulb of the evelash. $\times 60$
(3)

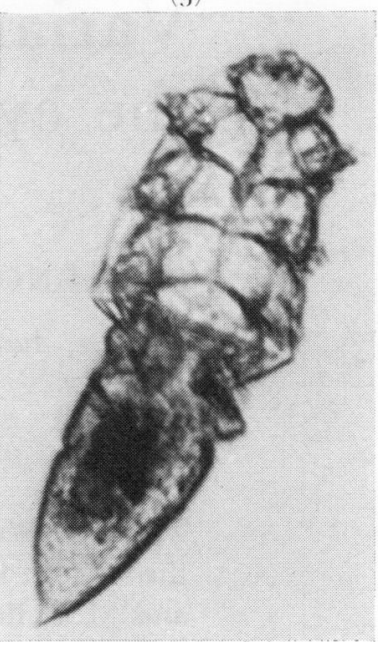

FIG. 3 Ventral view of caudate or short type of Demodex folliculorum.

Higher magnification (Fig. 3) revcals this variant to have an overall stumpy appearance with a short abdomen, characterized by a pointed caudal extremity.

The capitulum is mature in development and segmentation of the thorax is well defined. The characteristic annular banding of the abdominal wall is absent.

The overall body length is three times the width of the cephalothorax, whereas in the normal long type this may be seven times the width of the cephalothorax. The abdome was approximately the same length as the cephalothorax, whereas in the normal type, ing may be two and a half times as long as the prosoma. The short parasite was not as active as the larger form.

\section{Comment}

Demodectic mites enjoy a wide distribution throughout the animal kingdom, parasitizing the sebaceous glands and hair follicles of most mammals. The varieties encountered and their respective hosts include Demodex canis (dog), Demodex cati (cat), Demodex phylloides (pig), Demodex bovis (cattle), Demodex equi (horse), Demodex muscardini (dormouse), Demodex cuniculi (rabbit), Demodex ratti (rat), and numerous others. Much study has been devoted to differentiating these different species. The proportions of the body, the nature of the dorsal spines of the capitulum, the location of the male genitalia, and the structure of the ova are some of the important distinguishing characteristics of value to the acarologist.

When first observed the appearance of the caudate variety of Demodex folliculorum may be quite misleading, and it can readily be misidentified as Demodex phylloides which it closely resembles. Such a conclusion may lead one to rationalize, quite erroneously, that this feature confirms the disputed theory of cross-transmission of demodectic mites.

Demodex phylloides, which is responsible for demodectic mange in pigs, is a short acarine measuring up to $230 \mu$ in length, rather wide, with a total length about three and a half times the width of the cephalothorax. Like the caudate variety of Demodex folliculorum it ends in a narrow tail-like process. It differs in having a banded abdomen and the male genital orifice is located dorsally in a more anterior position between the first and second legs. 
Demodex equi may also be a source of confusion when the posterior end of the abdomen is bluntly pointed. Often, however, it is smoothly rounded off and presents no problem in differentiation. In passing, it is interesting to note that the affected patient enjoyed a variety of occupations true to the egalitarian background of Australian society. These ranged from whisky dealer, salesman, and company manager to horse trainer. In this last mentioned context the potential of the infesting organism was of particular interest.

According to Hirst (1919) we are indebted to the studies of Erasmus Wilson (1844), that astute lecturer in anatomy and physiology of the Middlesex Hospital, London, who was responsible for the elucidation and description of the caudate variety of Demodex folliculorum in the rigth century.

Shortly after Simon (1842) produced his paper on Demodex folliculorum, Wilson devoted his full energies to the study of this parasite which he preferred to call Entozoon folliculorum. In his exhaustive investigations he came across the short form which he was able to differentiate and classify. He concluded that, during development, the common type passes through an intermediate form which does not apply to the short variety. He draws an interesting analogy with a similar modification seen in the common earth worm.

Wilson's studies permit an exact differential diagnosis from other species particularly those found in swine and horses, and assist the ophthalmologist in recognizing the various forms of Demodex folliculorum.

I am indebted to Prof. William Nutting of the University of Massachusetts, Amherst, and to Dr. Robert Domrow, acarologist, Queensland Institute of Medical Research, for their assistance in helping to differentiate this organism, and to the Editor of The Medical Journal of Australia for permission to reproduce Fig. I .

\section{References}

coston, т. o. (1967) Trans. Amer. ophthal. Soc., 65, 36 I

english, f. P. (1969) Med. J. Aust., r, 1359

HIRST, A. (I919) Nat. Hist. (Lond.) "Studies on Acari". British Museum, London

simon, G. (1842) Arch. Anat. Physiol., 9, 2 1 8

sмiтh, s., and мcGulloch, c. (I969) Canad. 7. Ophthal., 4, 3

wilson, E. (1844) Philos. Trans. roy. Soc. (Lond.), p. 305 